S1 Fig. Species scenario-specific distribution change throughout New England, USA. Distribution change was projected for nine wildlife species between current (2010) conditions and each of the NELFP scenarios: A) Business-As-Usual, B) Connected Communities, C) Yankee Cosmopolitan, D) Go It Alone, and E) Growing Global. Maps display changes in species probability of occurrence, derived from simulated distribution maps for 2010 and 2060 (see [34,62] for more details). 


\section{A. American black bear}

A. Recent Trends

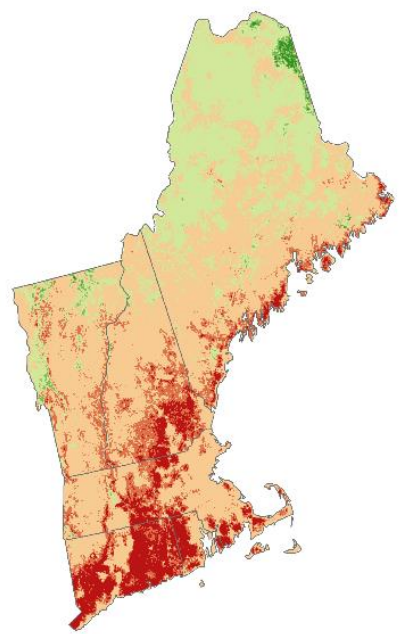

D. Go It Alone

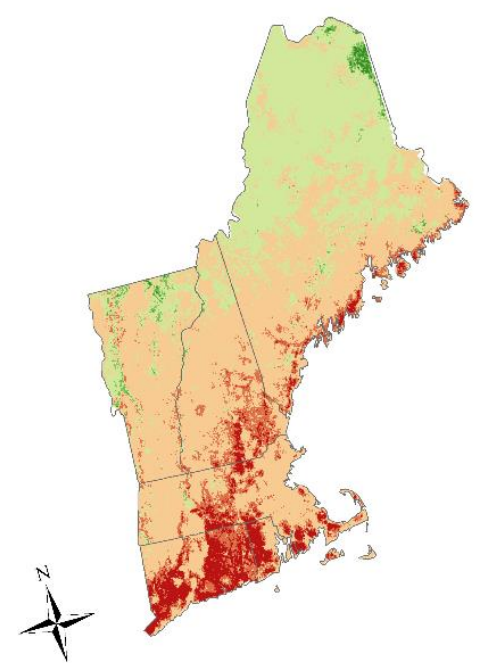

B. Connected Communities

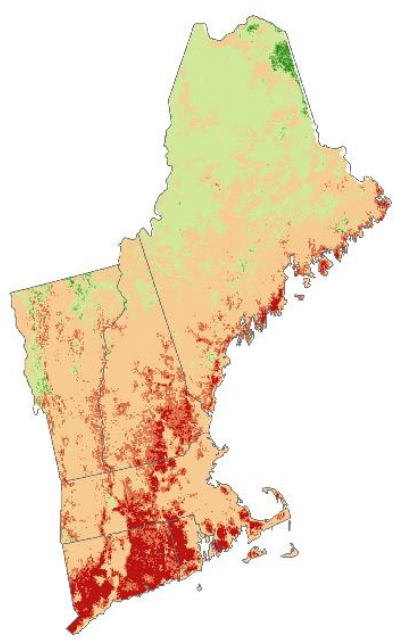

E. Growing Global

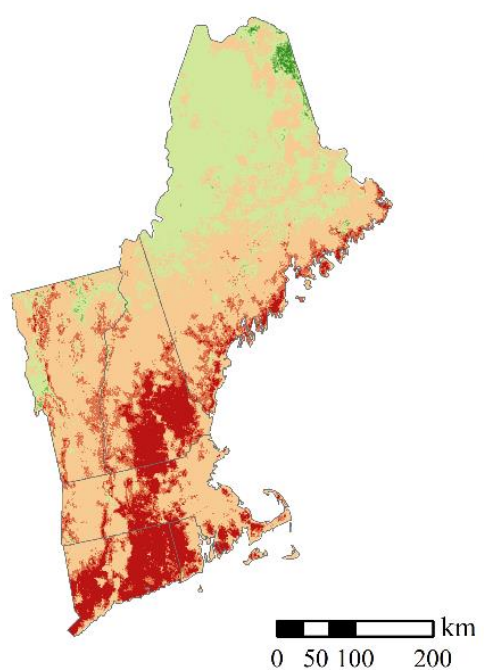

C. Yankee Cosmopolitan

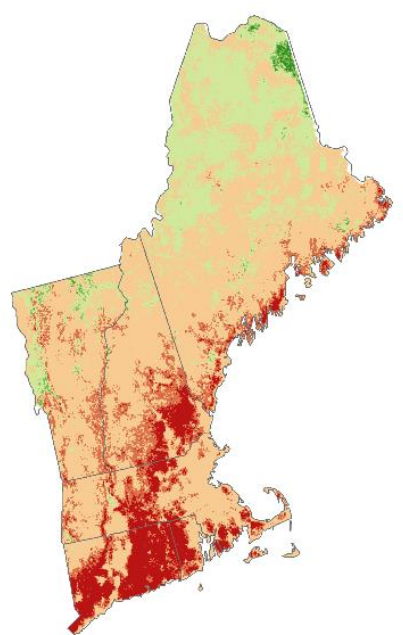

Change in Occurrence Probability

\begin{tabular}{l}
$-0.5--1$ \\
$-0.25--0.5$ \\
$0-0.25$ \\
$0-0.25$ \\
$0-25-0.5$ \\
$0.5-1$ \\
\hline$\square$
\end{tabular}




\section{B. Bobcat}

A. Recent Trends

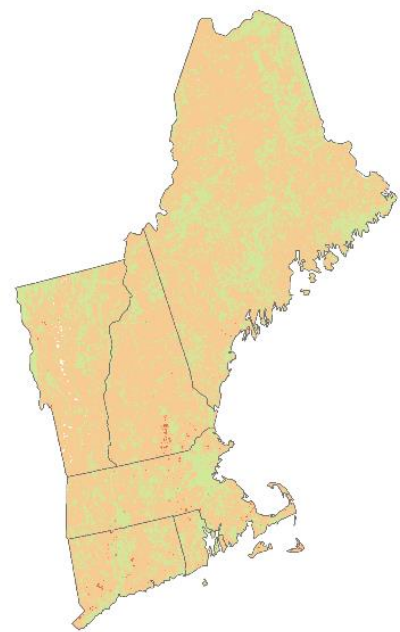

D. Go It Alone

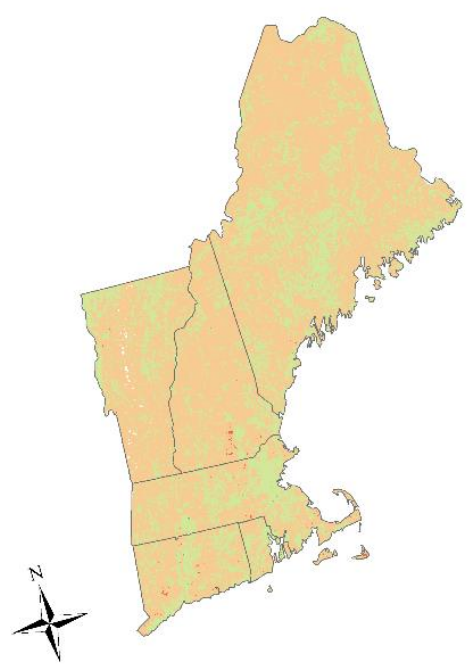

B. Connected Communities

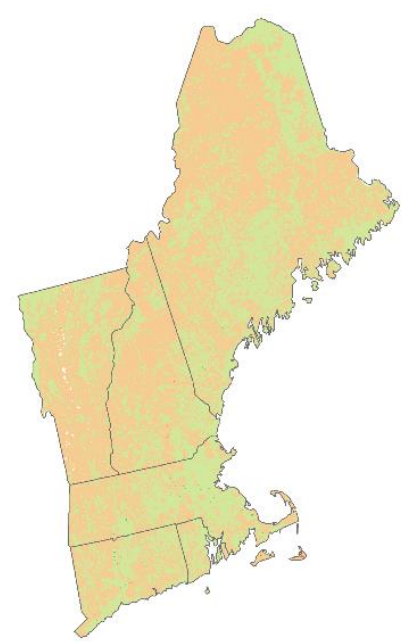

E. Growing Global

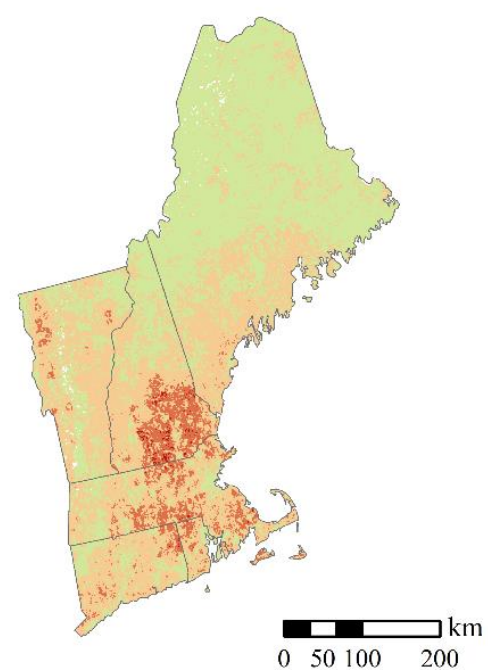

C. Yankee Cosmopolitan

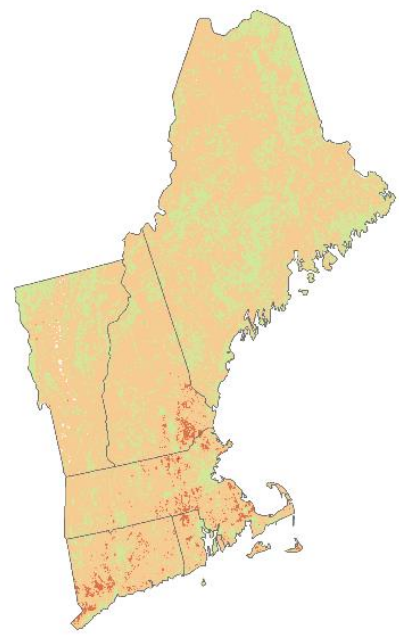

Change in Occurrence Probability

\begin{tabular}{l}
\hline \\
\hline \\
$\square$ \\
$-0.5--1$ \\
$\square-25--0.5$ \\
$0--0.25$ \\
$0-0.25$ \\
$0.25-0.5$ \\
$0.5-1$
\end{tabular}




\section{Coyote}

A. Recent Trends

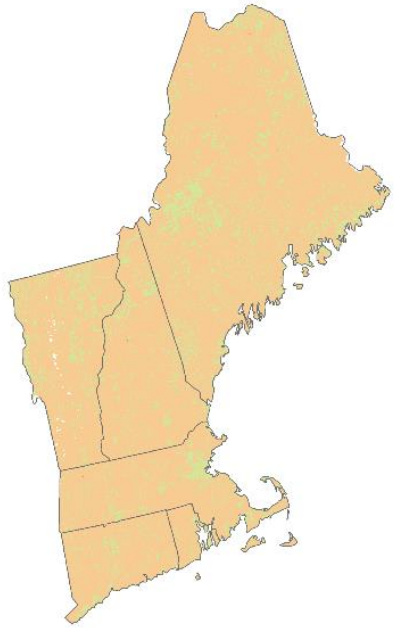

D. Go It Alone

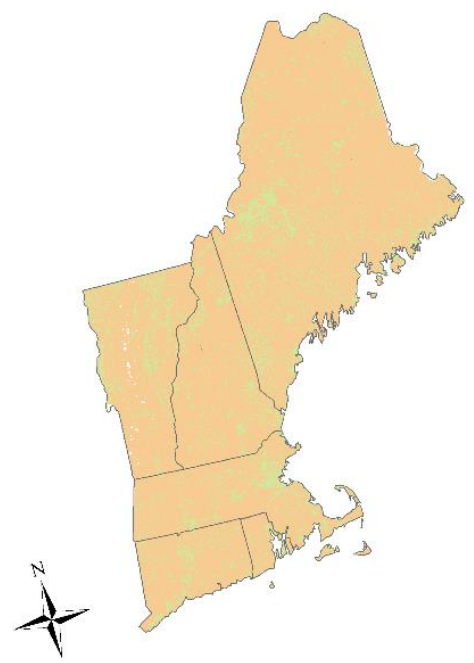

B. Connected Communities

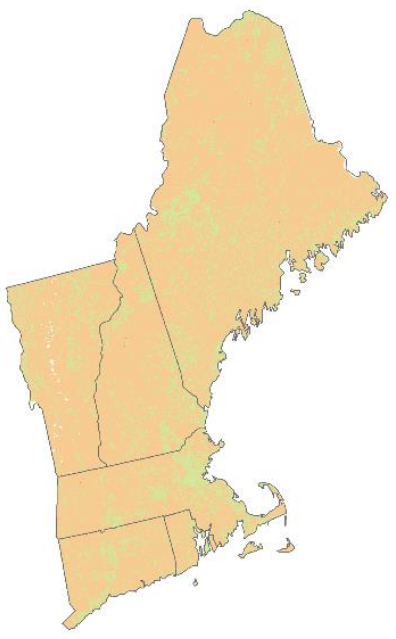

E. Growing Global

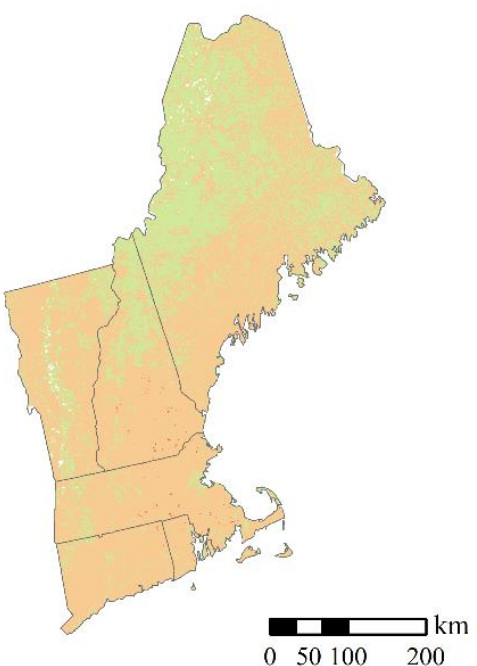

C. Yankee Cosmopolitan

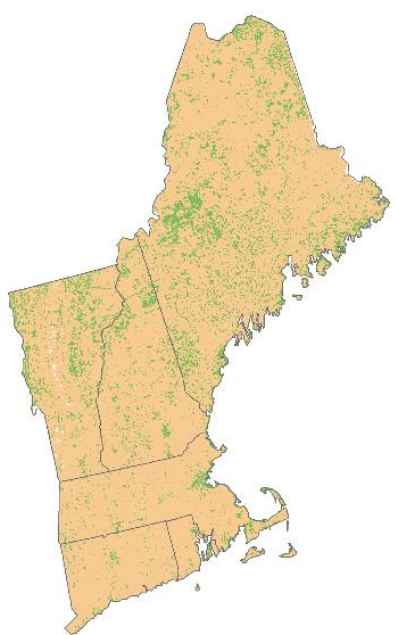

Change in Occurrence Probability

$-0.5--1$
$-0.25--0.5$
$0-0.25$
$0-0.25$
$0.25-0.5$
$0.5-1$




\section{Moose}

A. Recent Trends

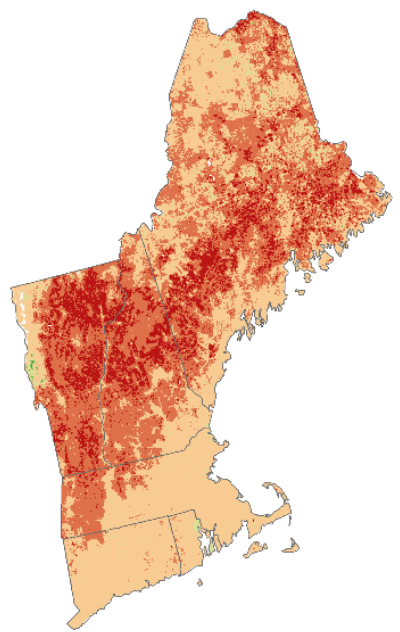

D. Go It Alone

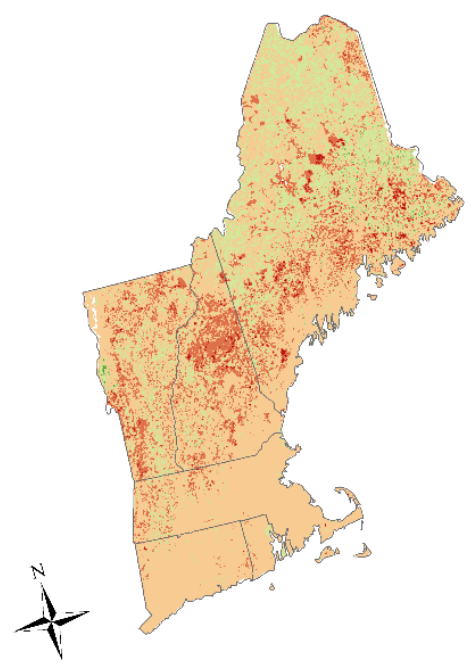

B. Connected Communities

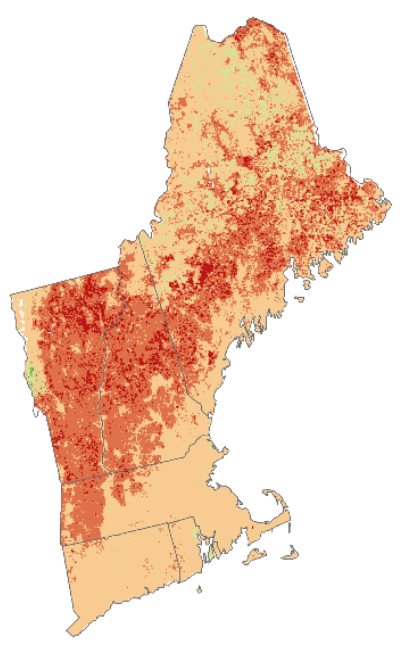

E. Growing Global

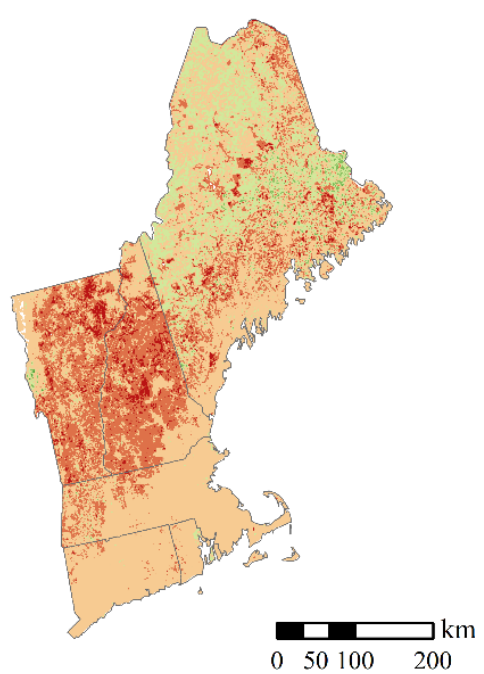

C. Yankee Cosmopolitan

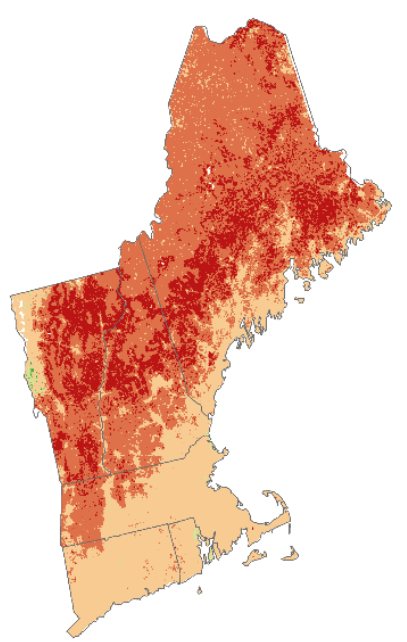

Change in Occurrence Probability

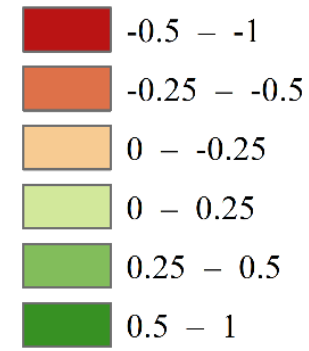




\section{E. Raccoon}

A. Recent Trends

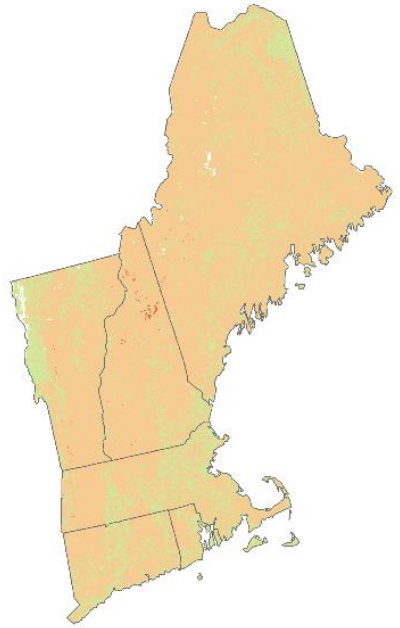

D. Go It Alone

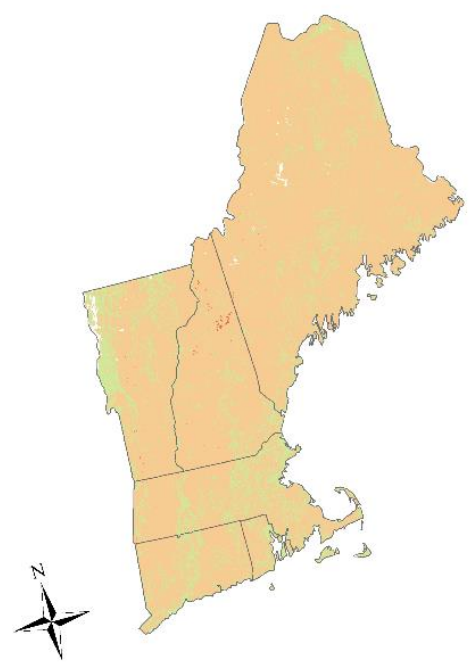

B. Connected Communities

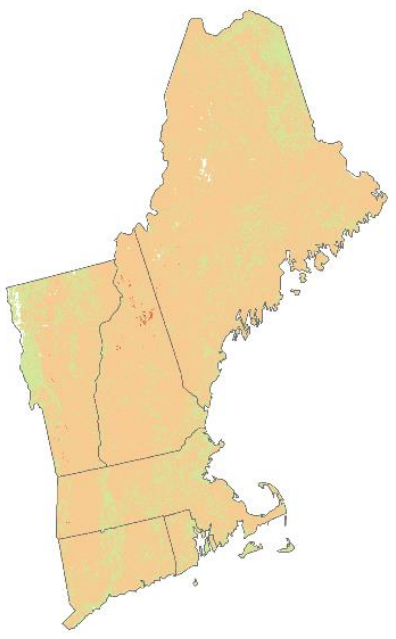

E. Growing Global

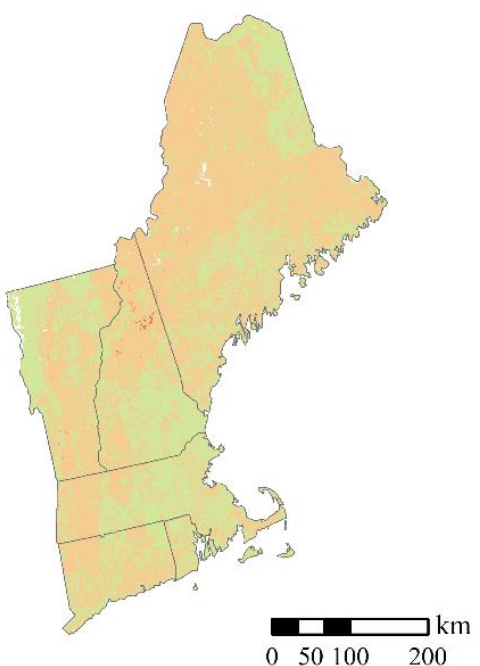

C. Yankee Cosmopolitan

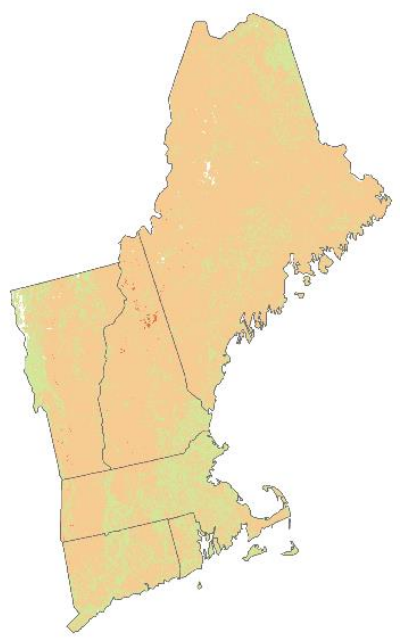

Change in Occurrence Probability

\begin{tabular}{l}
$-0.5--1$ \\
$-0.25--0.5$ \\
$0-0.25$ \\
$0-0.25$ \\
$0-25-0.5$ \\
$0.5-1$ \\
\hline$\square$
\end{tabular}




\section{F. Red fox}

A. Recent Trends

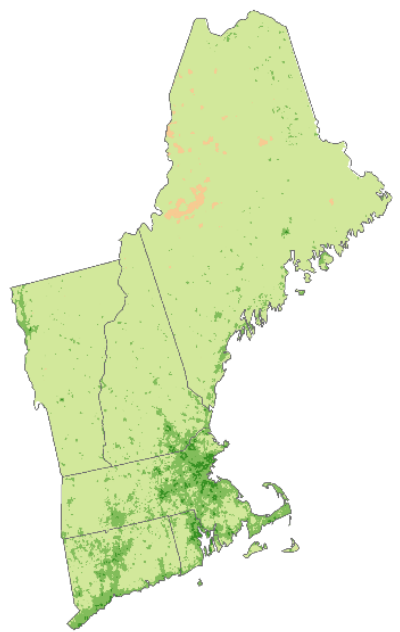

D. Go It Alone

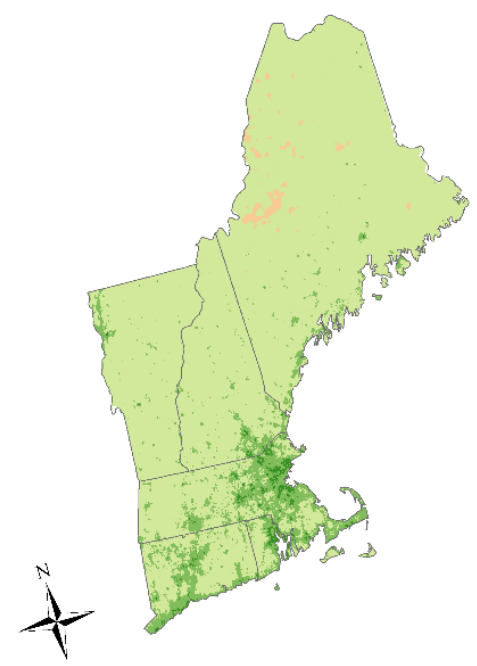

B. Connected Communities

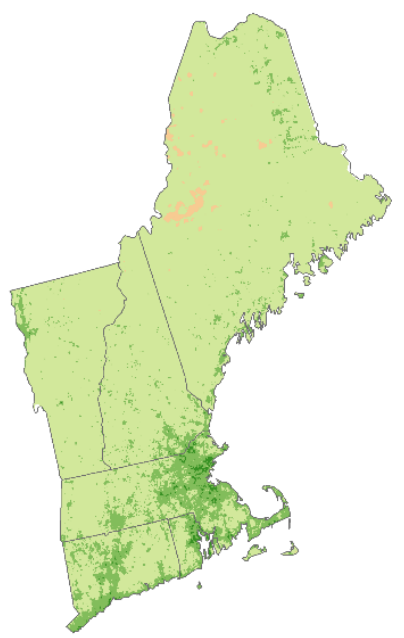

E. Growing Global

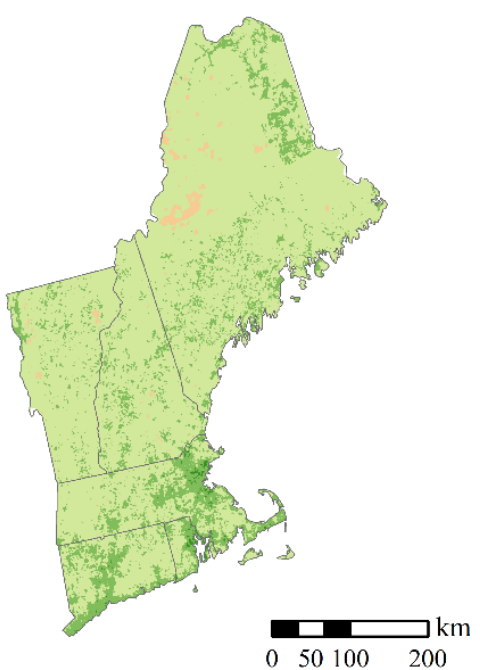

C. Yankee Cosmopolitan

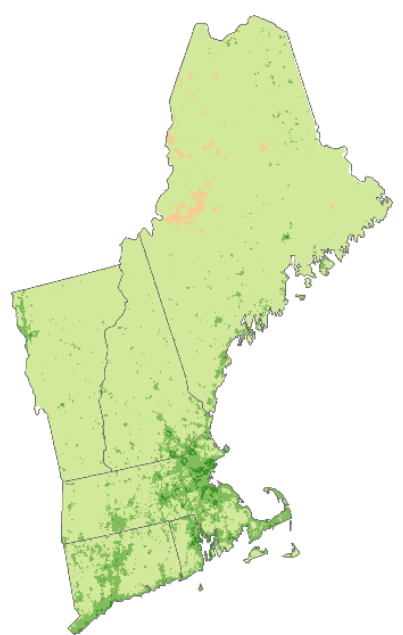

Change in Occurrence Probability

$-0.5--1$
$-0.25--0.5$
$0-0.25$
$0-0.25$
$0.25-0.5$
$0.5-1$




\section{G. Striped skunk}

A. Recent Trends

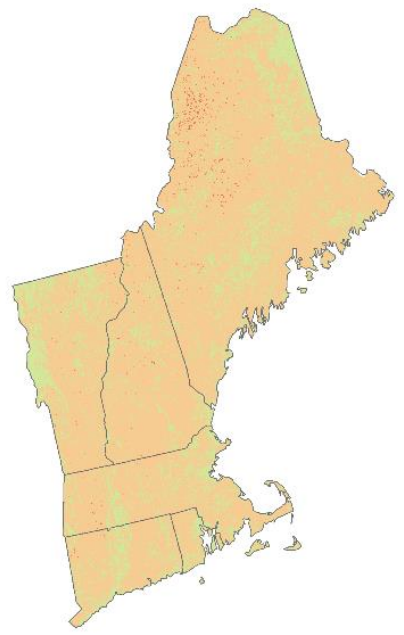

D. Go It Alone

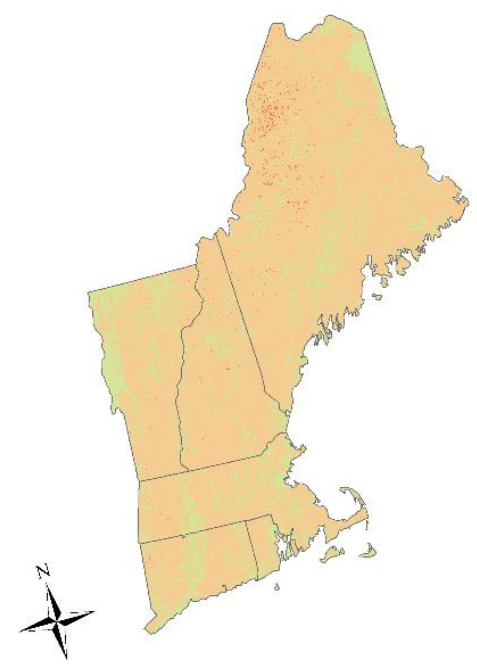

B. Connected Communities

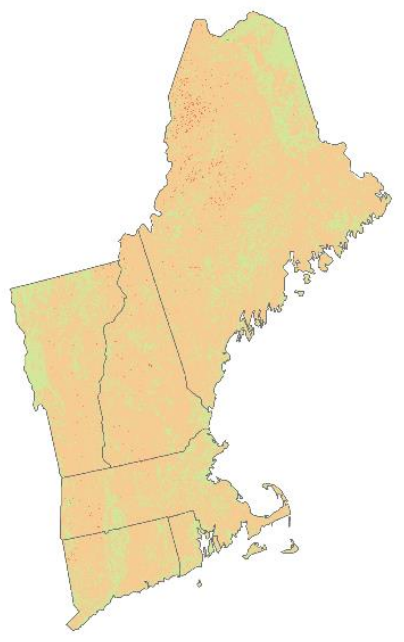

E. Growing Global

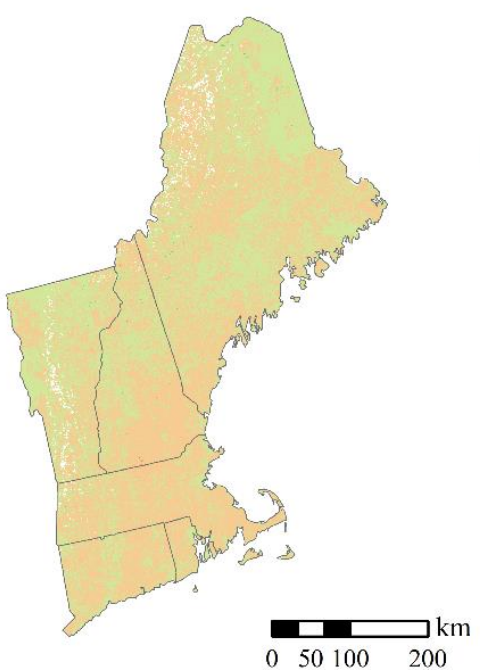

C. Yankee Cosmopolitan

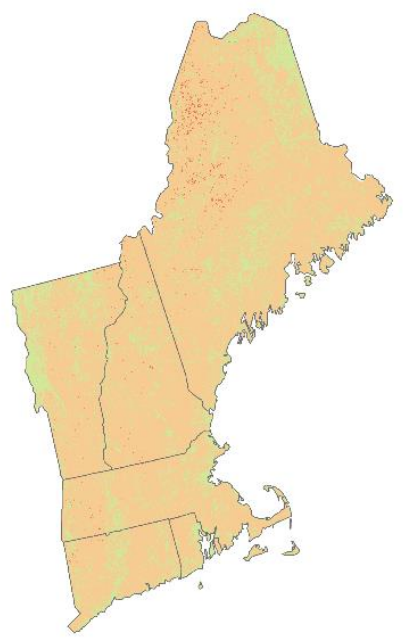

Change in Occurrence Probability

\begin{tabular}{l}
$-0.5--1$ \\
$-0.25--0.5$ \\
$0-0.25$ \\
$0-0.25$ \\
$0-25-0.5$ \\
$0.5-1$ \\
\hline$\square$
\end{tabular}




\section{H. White-tailed deer}

A. Recent Trends

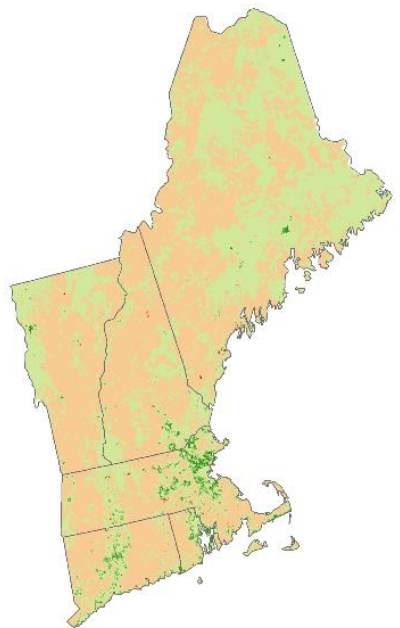

D. Go It Alone

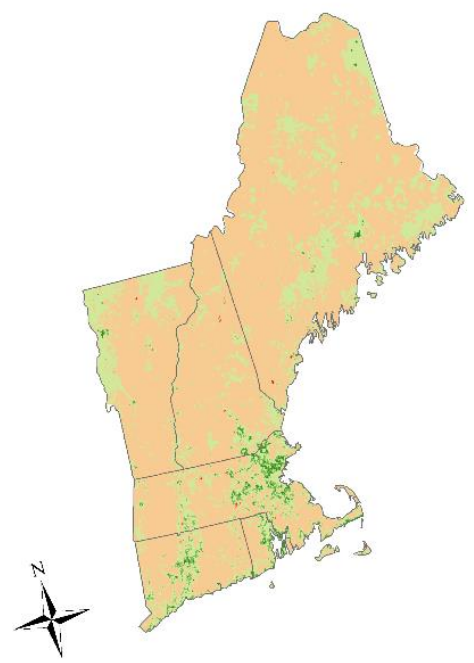

B. Connected Communities

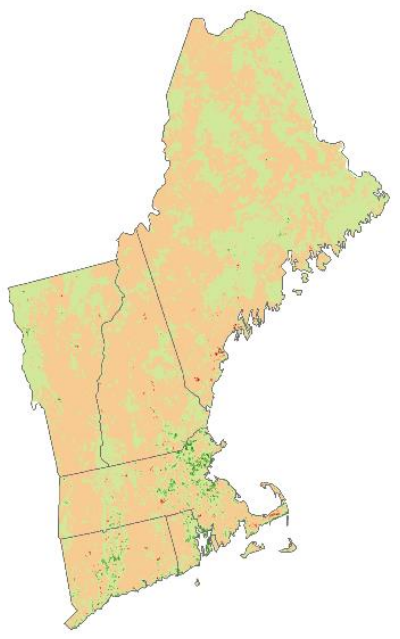

E. Growing Global

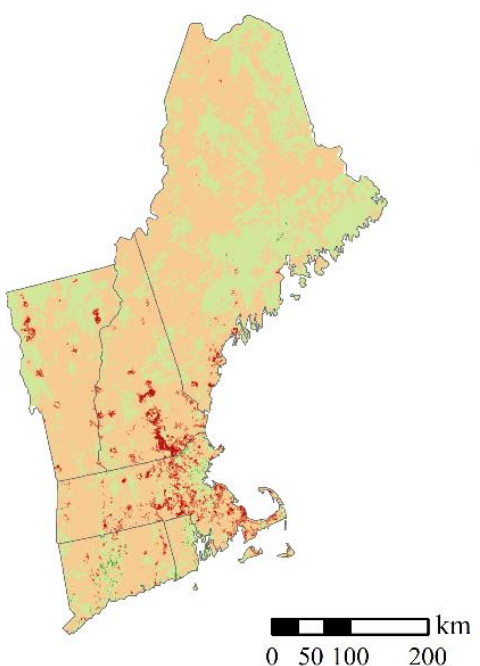

C. Yankee Cosmopolitan

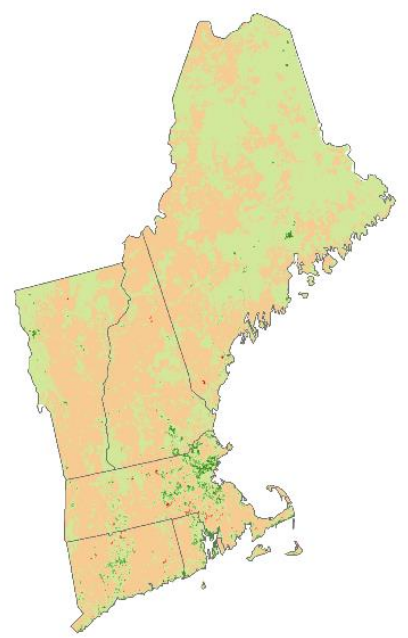

Change in Occurrence Probability

$$
\begin{aligned}
& \hline-0.5--1 \\
& -0.25--0.5 \\
& 0-0.25 \\
& 0-0.25 \\
& 0.25-0.5 \\
& \square-1 \\
& \hline \square
\end{aligned}
$$




\section{Wild turkey}

A. Recent Trends

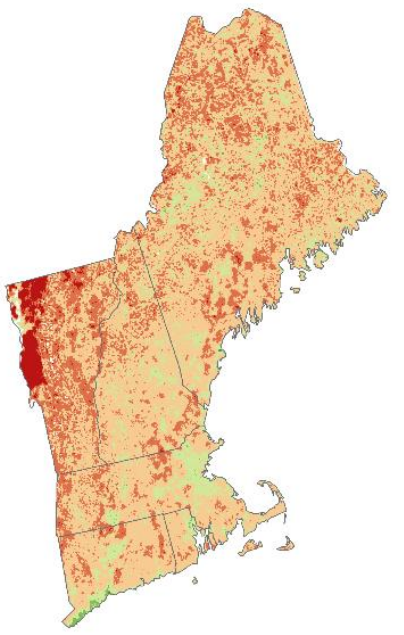

D. Go It Alone

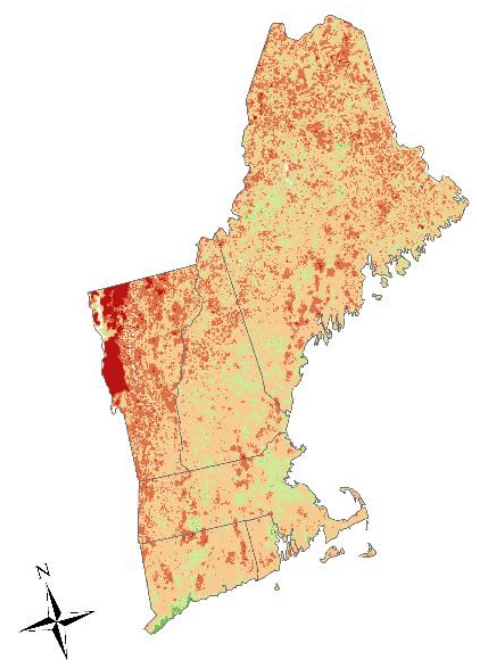

B. Connected Communities

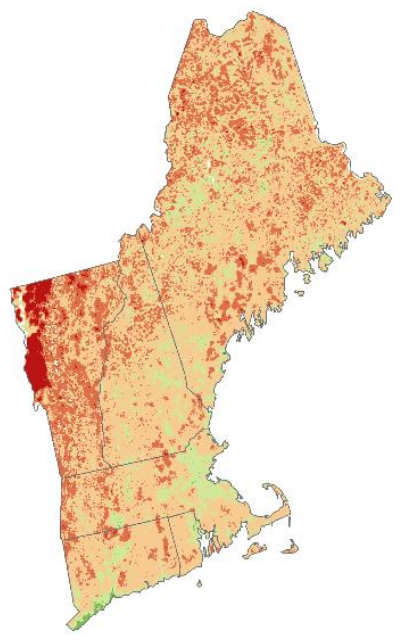

E. Growing Global

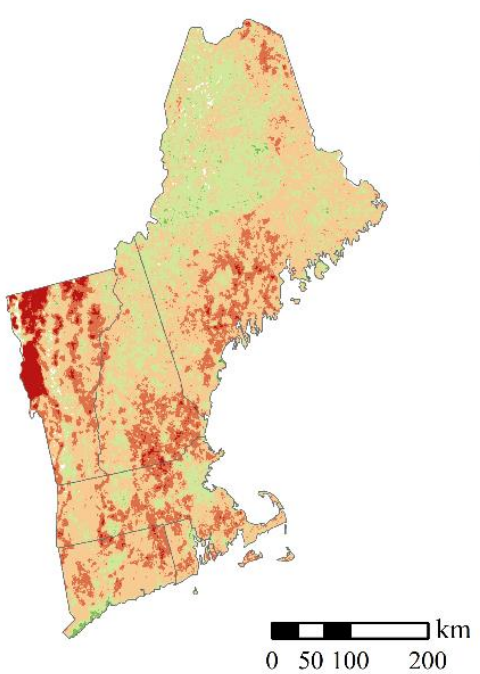

C. Yankee Cosmopolitan

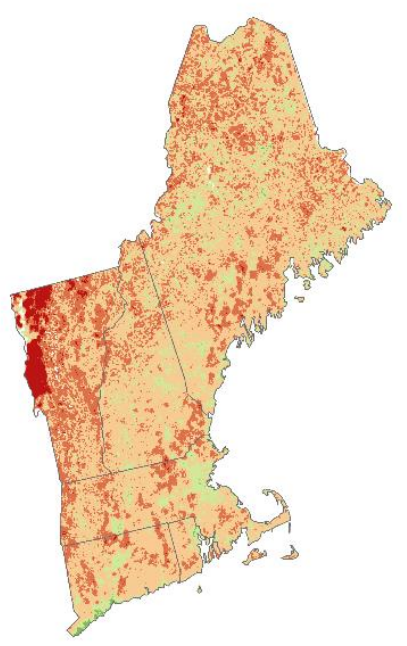

Change in Occurrence Probability

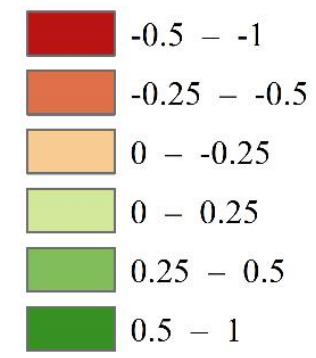

